RASĀYAN J. Chem.

Vol. 14 | No. 2 |887-892| April - June | 2021

ISSN: 0974-1496 | e-ISSN: 0976-0083 | CODEN: RJCABP

http://www.rasayanjournal.com

http://www.rasayanjournal.co.in

\title{
MICROWAVE ASSISTED CATALYST AND SOLVENT FREE EFFICIENT SYNTHESIS OF QUINOLINE DERIVATIVES BY THREE COMPONENT ONE POT AZA-DIELS-ALDER REACTION STRATEGY
}

\author{
Debajyoti Bhuyan \\ Department of Chemistry, Dergaon Kamal Dowerah College, Dergaon, Golaghat-785614, \\ (Assam) India \\ ${ }^{\square}$ Corresponding Author: debachem.bhuyan@gmail.com
}

\begin{abstract}
A series of quinoline derivatives have been synthesized via a microwave-assisted catalyst and solvent-free threecomponent one-pot aza-Diels-Alder reaction strategy from 2-naphthylamine, aldehyde and diethyl-2benzylidenemalonate. The Structures of all the synthesized compounds were confirmed by spectral and elemental analyses.
\end{abstract}

Keywords: Quinoline, Green Synthesis, Catalyst and Solvent-free, Microwave-assisted, Aza-Diels Alder Reaction RASĀYAN J. Chem., Vol. 14, No.2, 2021

\section{INTRODUCTION}

Over the years, the synthesis of quinoline-containing molecules has received much attention owing to its diverse biological and pharmaceutical activities such as antimalarial, antiasthmatic, antibacterial, antiinflammatory, antiplatelet and antihypertensive properties. ${ }^{1-3}$ Thus apart from the various conventional popular methods reported so far by Skraup ${ }^{4}$, Conrad-Limpach-Knorr ${ }^{5}$, Pfitzinger ${ }^{6}$, Friedlander ${ }^{7}$, and Combes $^{8}$ etc., the last decade witnessed significant advancement towards the synthesis of this important structure. ${ }^{9-15}$

Aza-Diel Alder reaction is a popular cycloaddition reaction designed to synthesize a wide range of sixmembered nitrogen-containing heterocycles of significant importance. It is a [4+2] cycloaddition reaction having a Nitrogen atom in either diene or dienophile. Chemist all over the globe has craftily designed different diene and dienophile to accomplish this [4+2] cycloaddition for its atom economy and relatively non generation of the side products. Generally, diene and dienophile were synthesized separately and subsequently reacted to generate 6-membered heterocycles, and normally designing such methodologies defy two prime aspects of Green Chemistry viz. minimization of the energy content and elimination or reduction of the use of toxic solvents in chemical transformation having a detrimental effect on the environment. In recent years microwave energy is used as a non-conventional source of energy which reduces the energy requirements of a reaction as well as considerably reduces the reaction time. Thus microwave coupled with the in-situ generation of either dienophile or diene or both carried out in absence of any solvent can be regarded as an impressive green replacement to the other widely accepted procedures. Because of the above facts, a study was undertaken to develop an efficient catalyst and solvent-free green synthetic methodology for the construction of quinoline derivatives. Thus in the present study, a microwave-assisted efficient method for the preparation of quinoline derivatives $\mathbf{4}$ via a catalyst and solvent-free three-component reactions of 2-naphthylamine 1,aromatic aldehyde 2, diethyl2-benzylidenemalonate $\mathbf{3}$ is described (Scheme-1).

\section{EXPERIMENTAL}

\section{Materials and Methods}

All the available reagents were purchased from Sigma Aldrich. These reagents are used without any further purification. Buchi M-560 melting point apparatus is used to record the melting points. IR spectra were recorded on a SHIMADZU FTIR-8400 instrument. ${ }^{1} \mathrm{H}$ and ${ }^{13} \mathrm{C}$ nuclear magnetic resonance (NMR) 
RASĀYAN J. Chem.

Vol. 14 | No. 2 |887-892| April - June | 2021

spectra were recorded on Brucker Avance DPX 300 MHz FT-NMR spectrometer and Brucker Advance DPX $75 \mathrm{MHz}$ FT-NMR spectrometer respectively. For ${ }^{1} \mathrm{H}$ NMR, Chemical shifts $(\delta)$ are given from Tetramethylsilane $(0 \mathrm{ppm})$ and coupling constants are expressed in $\mathrm{Hz}$. For ${ }^{13} \mathrm{C}$, Chemical shifts $(\delta)$ are given from $\mathrm{CDCl}_{3}(77.0 \mathrm{ppm})$. Mass spectra were recorded on ESQUIRE 3000 Mass spectrometer. All Microwave reactions were performed in an Anton Paar Synthos 3000 microwave reactor. All the reactions were monitored by thin-layer chromatography performed on pre-coated silica gel plates purchased from Merck. For UV active materials, the plate was visualized under UV illumination at 254 nm. Further visualization was accomplished by spraying $\mathrm{KMnO}_{4}$.

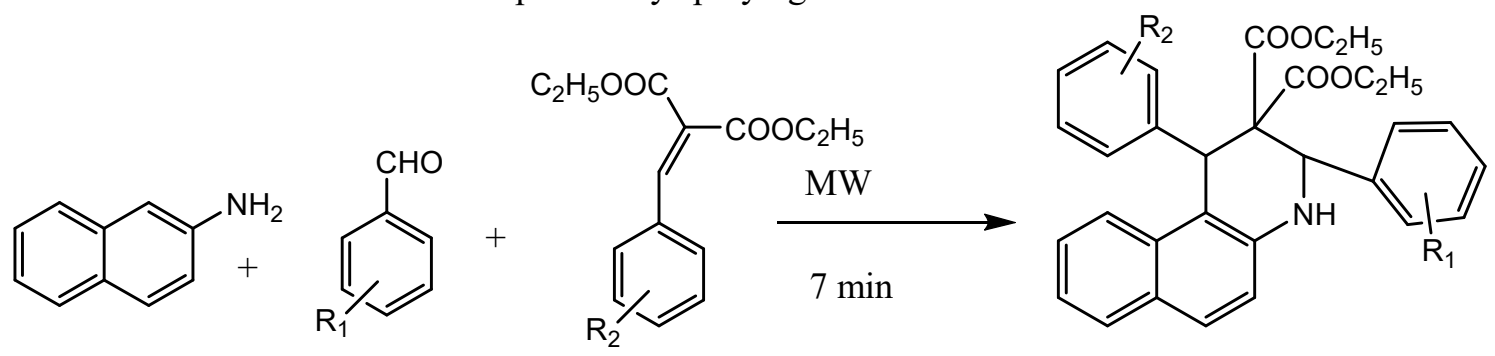

Scheme-1: Microwave-assisted Synthesis of Quinoline Derivatives under Solvent-free Condition

\section{General Procedure}

2-naphthylamine (1 mmol) 1, aromatic aldehyde $(1.5 \mathrm{mmol})$ 2, and diethyl-2-benzylidenemalonate 3 (1 mmol) were mixed in a reaction tube and irradiated at $720 \mathrm{~W}$ in a microwave reactor for 7 mins. The reaction is monitored with the help of TLC. After the completion of the reaction, $10 \mathrm{ml}$ ethanol is added and pure product is obtained by recrystallization from the water-ethanol mixture. Diethyl-2benzylidenemalonate is synthesized as per the standard procedure. ${ }^{16}$

\section{RESULTS AND DISCUSSION}

Initially, a three-component reaction was performed by reacting a mixture of 2-naphthylamine, benzaldehyde and diethyl-2-benzylidenemalonate in a microwave reactor (Anton Paar, Synthos 3000) in presence of methanol. When the reaction was carried out for 7 mins, quinoline derivatives formed at $25 \%$ yield. Therefore the progress of the reaction was studied in different organic solvents (Table-1, entries 19). It is observed that the reaction proceed efficiently absence of any solvent (Table-1, entry 5). Further, a negative result was observed when the reaction was carried out in an aqueous medium (Table-1, entry $10)$.

Table-1: Optimization Study of the Reaction in Different Solvents ${ }^{\mathrm{a}}$

\begin{tabular}{c|c|c|c}
\hline Entry & Solvent & Time & Yield (\%) \\
\hline 1 & Methanol & 7 & 25 \\
\hline 2 & Ethanol & 7 & 35 \\
\hline 3 & Acetonitrile & 7 & 12 \\
\hline 4 & DMSO & 7 & 12 \\
\hline 5 & No Solvent & 7 & 86 \\
\hline 6 & Nitrobenzene & 7 & 10 \\
\hline 7 & Tetrahydrofuran & 7 & 5 \\
\hline 8 & Toluene & 7 & 10 \\
\hline 9 & DMF & 7 & 2 \\
\hline 10 & Water & 7 & 0 \\
\hline
\end{tabular}

${ }^{\text {a Isolated yield }}$

To explore the synthetic scope of this reaction further, differently substituted aldehydes were employed under a similar set of conditions (Table-2). The reaction proceeds efficiently with electron-releasing or electron-withdrawing substituted benzaldehydes (78-88\% yields). Results are summarized in Table-2. Further, it was observed that no unwanted side reaction was formed and the required quinoline derivative was found as the sole product. The structures of all the synthesized compounds were confirmed by spectral analyses. 
RASĀYAN J. Chem.

Vol. 14 | No. 2 |887-892| April - June | 2021

It can be mechanistically justified that the observed products are formed via a formal [4+2] aza-DielsAlder pathway. ${ }^{17}$ The Schiff base generated in situ from naphthylamine $\mathbf{1}$ and the aldehyde $\mathbf{2}$ acts as a diene and thus reacts with the dienophile diethyl-2-benzylidenemalonate $\mathbf{3}$ to form the observed product 4 (Scheme-2). The product formation by [4+2] aza-Diels-Alder reaction pathway was further ascertained by carrying out the reaction stepwise. Firstly, imine $[\mathbf{A}]$ was prepared by stirring a mixture of naphthylamine $\mathbf{1}$ with benzaldehyde $\mathbf{2 a}$ at room temperature in ethanol. Finally, diethyl-2benzylidenemalonate $\mathbf{3}$ was reacted withthe imine [A] in a microwave reactor under catalyst and solventfree conditions to get the product $4 \mathbf{a}$.

Table-2: Generalization of the Reaction by Varying differently Substituted Aldehydes

\begin{tabular}{c|c|c|c|c}
\hline \\
\hline
\end{tabular}

${ }^{a}$ Isolated Yield

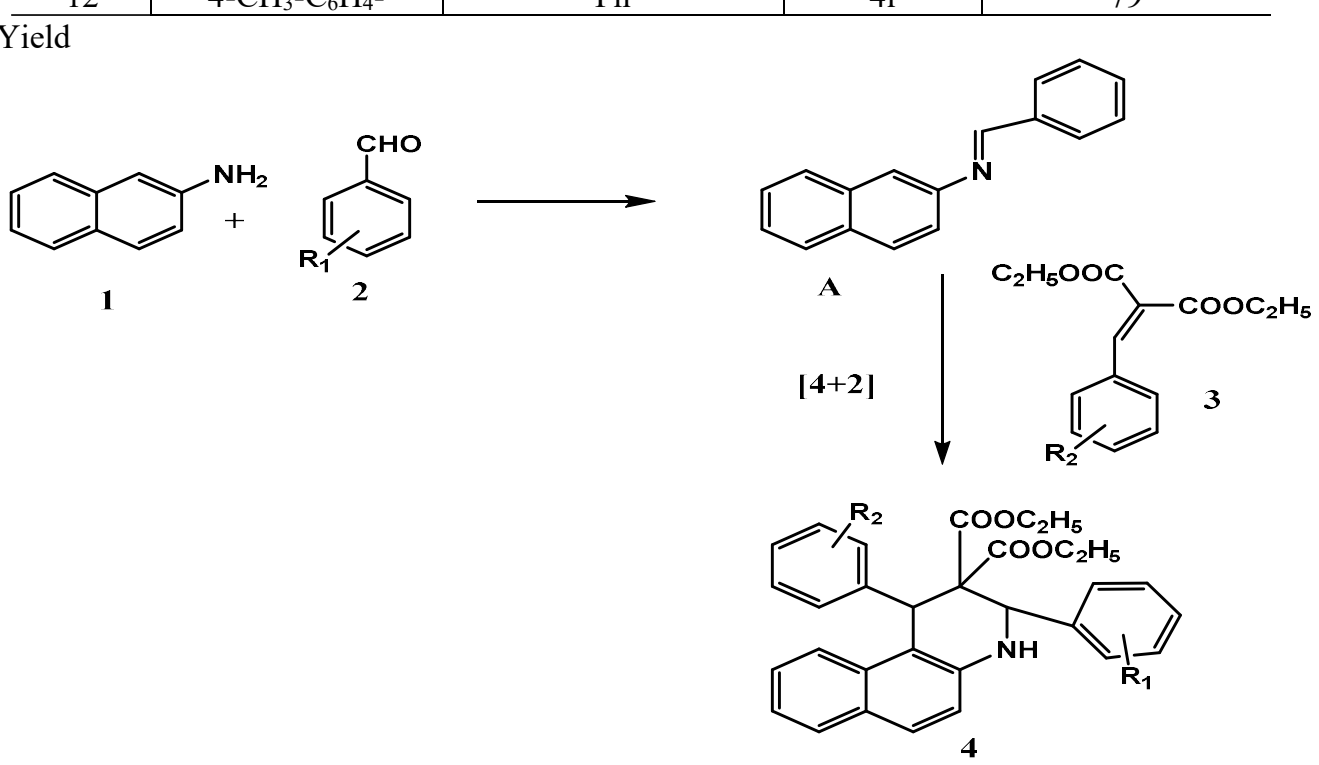

Scheme-2: Mechanism of Formation of Quinoline Derivative Via formal [4+2] Cycloaddition

\section{Spectral Data of the Synthesized Compounds}

Compound 4a: White solid m.p. 178-180 ${ }^{\circ} \mathrm{C},{ }^{1} \mathrm{H}$ NMR (300MHz, $\left.\mathrm{CDCl}_{3}\right) \delta$ 8.78-7.07 (m, Ar, 16H), 5.56 (s, 1H), $4.94(\mathrm{~s}, 1 \mathrm{H}), 4.72$ (s, br, 1H), 4.15 (q, 4H), $1.21(\mathrm{t}, 6 \mathrm{H}){ }^{13} \mathrm{C} \mathrm{NMR}\left(75 \mathrm{MHz}, \mathrm{CDCl}_{3}\right) \delta 172.5$, 
RASĀYAN J. Chem.

Vol. 14 | No. 2 |887-892| April - June | 2021

$155.1,149.7,138.2,133.9,132.9,132.6,130.6,128.8,128.7,128.5,125.4,123.7,120.3,118.8,117.2$, $116.3,72.2,62.3,60.7,38.4,20.1$; IR $\left(\mathrm{CHCl}_{3}, \mathrm{~cm}^{-1}\right) 3410,1736,1605,1509,1381,1229,755 . \mathrm{MS}$ (GCMS, m/z) 479.2 [M] ; Anal. Calculated for $\mathrm{C}_{31} \mathrm{H}_{29} \mathrm{NO}_{4}$ : C, 77.64; H, 6.10; N, 2.92. Found: C, 77.58; H, 5.94; N, 2.82 .

Compound 4b:White solid m.p. 174-176 ${ }^{\circ} \mathrm{C},{ }^{1} \mathrm{H}$ NMR $\left(300 \mathrm{MHz}, \mathrm{CDCl}_{3}\right) \delta$ 8.78-7.02 (m, Ar, 14H), 5.58 (s, 1H), $4.97(\mathrm{~s}, 1 \mathrm{H}), 4.73(\mathrm{~s}, \mathrm{br}, 1 \mathrm{H}), 4.13(\mathrm{q}, 4 \mathrm{H}), 1.22(\mathrm{t}, 6 \mathrm{H}){ }^{13} \mathrm{C}$ NMR $\left(75 \mathrm{MHz}, \mathrm{CDCl}_{3}\right) \delta 173.3$, $155.1,149.7,138.2,133.9,132.9,132.6,130.7,128.9,128.7,128.5,125.4,123.4,120.3,118.8,117.2$, 116.3, 72.2, 61.1, 60.1 36.4, 19.4; IR $\left(\mathrm{CHCl}_{3}, \mathrm{~cm}^{-1}\right) 3417,2955,1728,1623,1515,1390,1218,755 . \mathrm{MS}$ (GCMS, m/z) 637.1 [M] ; Anal. Calculated for $\mathrm{C}_{31} \mathrm{H}_{27} \mathrm{Br}_{2} \mathrm{NO}_{4}$ : C, 58.42; H, 4.27; N, 2.20. Found: C, $58.36 ; \mathrm{H}, 4.18 ; \mathrm{N}, 2.12$.

Compound 4c: White solid m.p. 189-191 ${ }^{\circ} \mathrm{C},{ }^{1} \mathrm{H}$ NMR $\left(300 \mathrm{MHz}, \mathrm{CDCl}_{3}\right) \delta$ 8.78-7.10 (m, Ar, 15H), 5.65 (s, 1H), $4.91(\mathrm{~s}, 1 \mathrm{H}), 4.75$ (s, br, 1H), $4.18(\mathrm{q}, 2 \mathrm{H}), 1.24$ (t, 3H), ${ }^{13} \mathrm{C}$ NMR $\left(75 \mathrm{MHz}, \mathrm{CDCl}_{3}\right) \delta 170.2$, $151.1,146.0,136.1$, , 132.5, 129.8, 127.4, 126.7, 126.1, 125.2, 124.1, 123.2, 120.2, 118.8, 117.1, 114.4, 70.2, 61.6, 60.2, 35.4, 19.8; IR $\left(\mathrm{CHCl}_{3}, \mathrm{~cm}^{-1}\right) 3412,1728,1623,1515,1390,1218, \mathrm{MS}(\mathrm{GCMS}, \mathrm{m} / \mathrm{z})$ $513.2[\mathrm{M}]^{+}$; Anal. Calculated for $\mathrm{C}_{31} \mathrm{H}_{28} \mathrm{ClNO}_{4}: \mathrm{C}, 72.44 ; \mathrm{H}, 5.49 ; \mathrm{N}, 2.73$. Found: $\mathrm{C}, 72.34 ; \mathrm{H}, 5.44 ; \mathrm{N}$, 2.62 .

Compound 4d: White solid m.p. 166-168 ${ }^{\circ} \mathrm{C},{ }^{1} \mathrm{H}$ NMR $\left(300 \mathrm{MHz}, \mathrm{CDCl}_{3}\right) \delta 8.78-7.17(\mathrm{~m}, \mathrm{Ar}, 14 \mathrm{H}), 5.56$ (s, 1H), $4.91(\mathrm{~s}, 1 \mathrm{H}), 4.75$ (s, br, 1H), 4.18 (q, 4H), 2.25. (s, 3H), 1.24 (t, 6H), ${ }^{13} \mathrm{C}$ NMR $(75 \mathrm{MHz}$, $\left.\mathrm{CDCl}_{3}\right) \delta 170.6,156.1,143.0,136.1,, 132.9,132.8,128.4,126.4,126.1,125.9,125.2,122.8,121.6$, 118.4, 117.6, 114.2, 69.2, 62.2, 58.1, 34.4, 29.8, 19.8; IR $\left(\mathrm{CHCl}_{3}, \mathrm{~cm}^{-1}\right) 3408,1734,1621,1501,1394$, 1221, MS (GCMS, m/z) 527.1 [M] ; Anal. Calculated for $\mathrm{C}_{32} \mathrm{H}_{30} \mathrm{ClNO}_{4}: \mathrm{C}, 72.79 ; \mathrm{H}, 5.73 ; \mathrm{N}, 2.65$. Found: C, 72.66; H, 5.68; N, 2.52 .

Compound 4e: pale yellow solid $168-170{ }^{\circ} \mathrm{C},{ }^{1} \mathrm{H}$ NMR $\left(300 \mathrm{MHz}, \mathrm{CDCl}_{3}\right) \delta$ 8.77-7.11 (m, Ar, $\left.14 \mathrm{H}\right)$, ), $5.58(\mathrm{~s}, 1 \mathrm{H}), 4.95(\mathrm{~s}, 1 \mathrm{H}), 4.78(\mathrm{~s}, \mathrm{br}, 1 \mathrm{H}), 4.11(\mathrm{q}, 4 \mathrm{H}), 1.22(\mathrm{t}, 6 \mathrm{H}),{ }^{13} \mathrm{C}$ NMR $\left(75 \mathrm{MHz}, \mathrm{CDCl}_{3}\right) \delta$ 173.9, 157.1, 142.7, 136.8, 133.2, 132.8, 132.2, 131.4, 129.1, 128.7, 127.2, 125.8, 123.6, 120.4, 118.5, 116.2, 114.3, 70.2, 61.6, 58.8, 35.4, 19.9; IR $\left(\mathrm{CHCl}_{3}, \mathrm{~cm}^{-1}\right) 3411,2977,1733,1625,1549,1389,1221$, MS (GCMS, m/z) 637.1 [M] $]^{+}$Anal. Calculated for $\mathrm{C}_{31} \mathrm{H}_{27} \mathrm{Br}_{2} \mathrm{NO}_{4}$ : C, 58.42; H, 4.27; N, 2.20. Found: C, $58.38 ; \mathrm{H}, 4.22 ; \mathrm{N}, 2.16$.

Compound 4f: Yellow solid 177-179 ${ }^{\circ} \mathrm{C},{ }^{1} \mathrm{H}$ NMR $\left(300 \mathrm{MHz}, \mathrm{CDCl}_{3}\right) \delta 8.79-7.05(\mathrm{~m}, \mathrm{Ar}, 14 \mathrm{H}), 5.62(\mathrm{~s}$, $1 \mathrm{H}), 5.04(\mathrm{~s}, 1 \mathrm{H}), 4.83$ (s, br, 1H), 4.38. (q, 4H), 1.32. (t, 6H), ${ }^{13} \mathrm{C}$ NMR $\left(75 \mathrm{MHz}, \mathrm{CDCl}_{3}\right) \delta$ 176.9, 157.1, $142.7,136.8,133.2,132.8,132.2,131.4,129.1,128.7,127.2,125.8,123.6,120.4,118.5,116.2,114.3$, 70.2, 61.9, 60.8, 35.8, 21.9; IR $\left(\mathrm{CHCl}_{3}, \mathrm{~cm}^{-1}\right) 3398,1733,1628,1541,1385,1241, \mathrm{MS}(\mathrm{GCMS}, \mathrm{m} / \mathrm{z})$ $569.1[\mathrm{M}]^{+}$; Anal. Calculated for $\mathrm{C}_{31} \mathrm{H}_{29} \mathrm{~N}_{2} \mathrm{O}_{8} \mathrm{C}, 65.37 ; \mathrm{H}, 4.78 ; \mathrm{N}, 7.38$. Found: C, 65.31; H, 4.68; N, 7.22

Compound 4g: yellow gum, ${ }^{1} \mathrm{H}$ NMR $\left(300 \mathrm{MHz}, \mathrm{CDCl}_{3}\right) \delta 8.78-7.13(\mathrm{~m}, \mathrm{Ar}, 14 \mathrm{H}), 5.56(\mathrm{~s}, 1 \mathrm{H}), 4.91(\mathrm{~s}$, $1 \mathrm{H}), 4.75(\mathrm{~s}, \mathrm{br}, 1 \mathrm{H}), 4.18(\mathrm{q}, 2 \mathrm{H}), 3.86 .(\mathrm{s}, 3 \mathrm{H}), 3.75 .(\mathrm{s}, 3 \mathrm{H}), 1.24(\mathrm{t}, 3 \mathrm{H}),{ }^{13} \mathrm{C}$ NMR $\left(75 \mathrm{MHz}, \mathrm{CDCl}_{3}\right)$ $\delta 170.6,151.4,139.8,137.8,136.8,134.3,132.7,132.5,131.8,130.1,129.7,128.5,128.0,126.7,126.4$, 125.1, 125.0, 123.8, 120.5, 120.2, 119.1, 118.2,69.2, 62.2, 58.1, 57.4, 57.2, 29.8, 19.8; IR $\left(\mathrm{CHCl}_{3}, \mathrm{~cm}^{-1}\right)$ 3412, 1734, 1621, 1501, 1394, 1221, MS (GCMS, m/z) $539.2[\mathrm{M}]^{+}$; Anal. Calculated for $\mathrm{C}_{33} \mathrm{H}_{33} \mathrm{NO}_{6} \mathrm{C}$, 73.45; H, 6.16; N, 2.60. Found: C, 73.33; H, 6.08; N, 2.52.

Compound 4h: pale yellow solid $168-170{ }^{\circ} \mathrm{C},{ }^{1} \mathrm{H}$ NMR $\left(300 \mathrm{MHz}, \mathrm{CDCl}_{3}\right) \delta$ 8.77-7.11 (m, Ar, $\left.15 \mathrm{H}\right)$, ), $5.58(\mathrm{~s}, 1 \mathrm{H}), 4.95(\mathrm{~s}, 1 \mathrm{H}), 4.78(\mathrm{~s}, \mathrm{br}, 1 \mathrm{H}), 4.11(\mathrm{q}, 2 \mathrm{H}), 1.22(\mathrm{t}, 3 \mathrm{H}),{ }^{13} \mathrm{C}$ NMR $\left(75 \mathrm{MHz}, \mathrm{CDCl}_{3}\right) \delta$ 173.9, 157.1, 142.7, 136.8, 133.2, 132.8, 132.2, 131.4, 129.1, 128.7, 127.2, 125.8, 123.6, 120.4, 118.5, 116.2, 114.3, 70.2, 61.6, 58.8, 35.4, 19.9; IR $\left(\mathrm{CHCl}_{3}, \mathrm{~cm}^{-1}\right) 3422,1733,1625,1549,1389,1221$, MS 
RASĀYAN J. Chem.

Vol. 14 | No. 2 |887-892| April - June | 2021

(GCMS, m/z) 557.1 [M] $]^{+}$. Anal. Calculated for $\mathrm{C}_{31} \mathrm{H}_{28} \mathrm{BrNO}_{4} \mathrm{C}, 66.67 ; \mathrm{H}, 5.05 ; \mathrm{N}, 2.51$. Found: $\mathrm{C}, 66.39$; $\mathrm{H}, 4.88 ; \mathrm{N}, 2.48$

Compound 4i: Yellow solid 177-179 ${ }^{\circ} \mathrm{C},{ }^{1} \mathrm{H}$ NMR $\left(300 \mathrm{MHz}, \mathrm{CDCl}_{3}\right) \delta 8.79-7.05(\mathrm{~m}, \mathrm{Ar}, 15 \mathrm{H}), 5.62(\mathrm{~s}$, $1 \mathrm{H}), 5.05(\mathrm{~s}, 1 \mathrm{H}), 4.85(\mathrm{~s}, \mathrm{br}, 1 \mathrm{H}), 4.38$. (q, $2 \mathrm{H}), 1.32$. (t, 3H), ${ }^{13} \mathrm{C}$ NMR $\left(75 \mathrm{MHz}, \mathrm{CDCl}_{3}\right) \delta 173.9,157.1$, 142.7, 136.8, 133.2, 132.8, 132.2, 131.4, 129.1, 128.7, 127.2, 126.8, 123.8, 122.4, 118.5, 116.2, 114.1, 70.4, 61.9, 60.8, 35.8, 21.3; IR $\left(\mathrm{CHCl}_{3}, \mathrm{~cm}^{-1}\right) 3421,1733,1628,1541,1385,1241, \mathrm{MS}(\mathrm{GCMS}, \mathrm{m} / \mathrm{z})$ $497.2[\mathrm{M}]^{+}$. Anal. Calculated for $\mathrm{C}_{31} \mathrm{H}_{28} \mathrm{FNO}_{4} \mathrm{C}, 74.83 ; \mathrm{H}, 5.67 ; \mathrm{N}, 2.82$. Found: C, 74.79; H, 5.58; N, 2.78

Compound 4j: White solid; mp 182-184 ${ }^{\circ} \mathrm{C} ;{ }^{1} \mathrm{H}$ NMR $\left(300 \mathrm{MHz}, \mathrm{CDCl}_{3}\right) \delta$ 7.90-7.78 (m, Ar, $\left.2 \mathrm{H}\right)$, 7.49$6.68(\mathrm{~m}, \mathrm{Ar}, 12 \mathrm{H}), 5.30(\mathrm{~s}, 1 \mathrm{H}), 5.10(\mathrm{~s}, 1 \mathrm{H}), 4.82(\mathrm{~s}, \mathrm{br}, 1 \mathrm{H}), 4.37(\mathrm{q}, 2 \mathrm{H}), 2.34(\mathrm{~s}, 3 \mathrm{H}), 2.29(\mathrm{~s}, 3 \mathrm{H})$; $1.29(\mathrm{t}, 3 \mathrm{H}){ }^{13} \mathrm{C} \mathrm{NMR}\left(75 \mathrm{MHz}, \mathrm{CDCl}_{3}\right) \delta 169.9,164.2,151.2,139.4,138.2,137.4,134.9,132.9,1328$, $131.1,130.4,129.8,128.3,128.1,126.5,126.3,125.4,124.8,123.3,120.8,120.3,120.1,118.6,63.2$, 56.4, 51.3, 26.4, 25.2, 21.2, 21.1; IR (thin film, $\mathrm{CHCl}_{3} \mathrm{~cm}^{-1}$ ) 3403, 1736, 1685, 1603, 1506, 1328, 779; MS (GCMS, $m / z) 507.2[\mathrm{M}]^{+}$. Anal. Calculated for $\mathrm{C}_{33} \mathrm{H}_{33} \mathrm{NO}_{4} \mathrm{C}, 78.08 ; \mathrm{H}, 6.55 ; \mathrm{N}, 2.76$. Found: C, $78.01 ; \mathrm{H}, 6.48 ; \mathrm{N}, 2.70$

Compound 4k: White solid; mp 158-160 ${ }^{\circ} \mathrm{C} ;{ }^{1} \mathrm{H}$ NMR (300MHz, $\left.\mathrm{CDCl}_{3}\right) \delta$ 7.88-6.88 (m, Ar, 15H), 5.26 $(\mathrm{s}, 1 \mathrm{H}), 5.05(\mathrm{~s}, 1 \mathrm{H}), 4.84(\mathrm{~s}, \mathrm{br}, 1 \mathrm{H}), 4.37(\mathrm{q}, 4 \mathrm{H}), 3.84(\mathrm{~s}, 3 \mathrm{H}), 1.29(\mathrm{t}, 6 \mathrm{H}){ }^{13} \mathrm{C} \mathrm{NMR}\left(75 \mathrm{MHz}, \mathrm{CDCl}_{3}\right)$ $\delta 169.9,164.2,151.2,139.4,138.2,137.4,134.9,132.9,1328,131.1,130.4,129.8,128.3,128.1,126.5$, $126.3,125.4,124.8,123.3,120.8,120.3,120.1,118.6,63.2,63.1,56.4,51.3,26.4,21.1$; IR (thin film, $\mathrm{CHCl}_{3} \mathrm{~cm}^{-1}$ ) 3408, 1739, 1613, 1516, 1321, 772; MS (GCMS, m/z) 509.2 [M] ${ }^{+}$. Anal. Calculated for $\mathrm{C}_{32} \mathrm{H}_{31} \mathrm{NO}_{5} \mathrm{C}, 75.42 ; \mathrm{H}, 6.13 ; \mathrm{N}, 2.75$. Found: $\mathrm{C}, 75.31 ; \mathrm{H}, 6.08 ; \mathrm{N}, 2.68$

Compound 4l: White solid; mp 152-154 ${ }^{0} \mathrm{C} ;{ }^{1} \mathrm{H}$ NMR (300MHz, $\left.\mathrm{CDCl}_{3}\right) \delta$ 7.88-6.88 (m, Ar, 15H), 5.26 $(\mathrm{s}, 1 \mathrm{H}), 5.05(\mathrm{~s}, 1 \mathrm{H}), 4.84(\mathrm{~s}, \mathrm{br}, 1 \mathrm{H}), 4.37(\mathrm{q}, 4 \mathrm{H}), 2.34(\mathrm{~s}, 3 \mathrm{H}), 1.29(\mathrm{t}, 6 \mathrm{H}){ }^{13} \mathrm{C} \mathrm{NMR}\left(75 \mathrm{MHz}, \mathrm{CDCl}_{3}\right)$ $\delta 169.9,164.2,151.2,139.4,138.2,137.4,134.9,132.9,132.8,131.1,130.4,129.8,128.3,128.1,126.5$, $126.3,125.4,124.8,123.3,120.8,120.3,120.1,118.6,63.2,56.4,51.3,26.4,25.8$ 21.1; IR (thin film, $\mathrm{CHCl}_{3} \mathrm{~cm}^{-1}$ ) 3409, 1731, 1601, 1526, 1329, 778; MS (GCMS, m/z) 593.2 [M] $]^{+}$, Anal. Calculated for $\mathrm{C}_{32} \mathrm{H}_{31} \mathrm{NO}_{4} \mathrm{C}, 77.87 ; \mathrm{H}, 6.33 ; \mathrm{N}, 2.84$. Found: $\mathrm{C}, 77.81 ; \mathrm{H}, 6.28 ; \mathrm{N}, 2.72$

\section{CONCLUSION}

In summary, an efficient and economical reaction methodology has been successfully demonstrated for synthesizing some complex quinoline derivatives via a microwave-assisted one-pot three-component azaDiels-Alder reaction strategy under catalyst and solvent-free conditions. This methodology can ease up serious problems like prolonged reaction time, stringent work up procedure and requirement of multi-step synthesis associated with other commonly accepted procedures. Further, higher atom economy, procedural simplicity and excellent yields make this effort very fascinating towards the synthesis of the quinoline derivatives.

\section{ACKNOWLEDGEMENT}

The author is thankful to Principal Dr. Ranjit Kr. Bordoloi for his support and constant encouragement.

\section{REFERENCES}

1. R.H. Manske, Chemical Reviews, 30(1), 113(1942), DOI:10.1021/cr60095a006

2. P.G. Bray, S.A. Ward, and P.M. O'Neil, Current Topics in Microbiology and Immunology, 295, 3(2005), DOI: 10.1007/3-540-29088-5_1

3. J. Achan, A.O. Talisuna, A. Erhart, A. Yeka, J.K.Tibenderana, F.N. Baliraine, P.J. Rosenthal, and U.D'. Alessandro, Malaria Journal, 10, 144(2011), DOI:10.1186/1475-2875-10-144

4. R.H.F. Manske, and M. Kukla, Organic Reaction,7, 59(1953), DOI:10.1002/0471264180.or007.02

5. R.H. Reitsema, Chemical Reviews, 43(1), 47(1948), DOI:10.1021/cr60134a002 
RASĀYAN J. Chem.

Vol. 14 | No. 2 |887-892| April - June | 2021

6. W. Pfitzinger, Journal Fürpraktische Chemie, 38(1), 582(1888), DOI:10.1002/prac.18880380138

7. J. S. Yadav, B.V.S. Reddy, P. Sreedhar, R.S. Rao, and K. Nagaiah, Synthesis, 2381(2004), DOI:10.1055/s-2004-831185

8. J.L. Born, Journal of Organic Chemistry, 37(24), 3952(1972), DOI:10.1021/j000797a045

9. J. Nan, Y. Hu, P. Chen, Y. Ma, Organic Letters, 21(7), 1984(2019), DOI:10.1021/acs.orglett.9b00039

10. S. Das, S. Sinha, D. Samanta, R. Mondal, G. Chakraborty, P. Brandaõ, and N.D. Paul, Journal of Organic Chemistry, 84(5), 10160(2019), DOI:10.1021/acs.joc.9b01343

11. Y. Men, J. Dong, S. Wang, and X. Xu, Organic Letters, 19(19), 6712(2017), DOI: 10.1021 /acs.orglett.7b03434

12. S.Y. Lee, and C.-H. Cheon, Journal of Organic Chemistry, 83(21), 13036(2018), DOI:10.1021/acs.joc.8b01675

13. R. Wang, H. Fan, W. Zhao, and F. Li, Organic Letters, 18(15), 3558(2016), DOI:10.1021/acs.orglett.6b01518

14. Q. Gao, S. Liu, X. Wu and A. Wu, Organic Letters, 16(17), 4582(2014), DOI:10.1021/ol502134u

15. H. Hegde, S.L. Gaonkar, N.P. Badiger and N.S. Shetty, Rasayan Journal of Chemistry, 13(3), 1744(2020), DOI:10.31788/RJC.2020.1335669

16. M.L. Deb and P.J. Bhuyan, Tetrahedron Letters, 46, 6453(2005), DOI:10.1016/j.tetlet.2005.07.111

17. D. Bhuyan, R. Sarma, Y. Dommaraju and D. Prajapati, Green Chemistry, 16, 1158(2014), DOI:10.1039/C3GC42389A

[RJC-6216/2020] 